\title{
PEROV TYPE RESULTS IN GAUGE SPACES AND THEIR APPLICATIONS TO INTEGRAL SYSTEMS ON SEMI-AXIS
}

\author{
Adela Novac* — Radu Precup** \\ (Communicated by Emanuel Chetcuti)
}

\begin{abstract}
In this paper we present Perov type fixed point theorems for contractive mappings in Gheorghiu's sense on spaces endowed with a family of vectorvalued pseudo-metrics. Applications to systems of integral equations are given to illustrate the theory. The examples also prove the advantage of using vectorvalued pseudo-metrics and matrices that are convergent to zero, for the study of systems of equations.
\end{abstract}

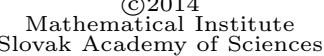

\section{Introduction and preliminaries}

In this paper we are concerning with the solvability of the semilinear operator system

$$
\left\{\begin{array}{l}
A(x, y)=x \\
B(x, y)=y
\end{array}\right.
$$

in a complete gauge space $X$ (space endowed with a family of pseudo-metrics). Here $A, B: X^{2} \rightarrow X$ are given nonlinear operators. Systems of this type arise from mathematical modelling of many interaction, competitive and cooperative processes from a variety of disciplines, including physics, biology, chemistry, engineering and other sciences. For instance, the system

$$
\left\{\begin{array}{l}
x(t)=\int_{t-\tau_{1}}^{t} f\left(s, x\left(\sigma_{1}(s)\right), y\left(\sigma_{2}(s)\right)\right) \mathrm{d} s \\
y(t)=\int_{t-\tau_{2}}^{t} g\left(s, x\left(\sigma_{1}(s)\right), y\left(\sigma_{2}(s)\right)\right) \mathrm{d} s
\end{array}\right.
$$

2010 Mathematics Subject Classification: Primary 47H10, 45G15; Secondary 47J05. Keywords: integral equation, gauge space, fixed point.

The second author was supported by a grant of the Romanian National Authority for Scientific Research, CNCS - UEFISCDI, project number PN-II-ID-PCE-2011-3-0094. 
is a mathematical model for the spread of two interacted infectious diseases with contact rates that vary seasonally. In these equations $x(t), y(t)$ represent the proportion of infectives in a population at time $t$, for each of the two epidemics; $\tau_{1}, \tau_{2}$ stand for the length of time an individual remains infectious of each one of the diseases; and $f, g$ are the proportion of new infectives per unit time for the two epidemics. The modified arguments $\sigma_{1}(t), \sigma_{2}(t)$ can be of retarded type, when $\sigma_{1}(t), \sigma_{2}(t) \leq t$, or of advanced type, if $\sigma_{1}(t), \sigma_{2}(t) \geq t$. For only one disease, and without argument deviations, such a model was introduced by Cooke and Kaplan 4] (see also Precup [12]).

It is obvious that system (1.1) can be viewed as a fixed point problem,

$$
T(u)=u
$$

in the space $X^{2}$, where $u=(x, y)$ and $T=(A, B)$. Therefore, we may think to apply to (1.3) , in $X^{2}$ endowed with the gauge structure induced by that of $X$, different abstract existences results from the theory of nonlinear operators on gauge spaces. Such a result is the contraction principle extended to gauge spaces by Colojoara (1961) [3], Gheorghiu (1967) [7] and Tarafdar (1974) [16]. However, as pointed out by Perov and Kibenko [1] in connection with Banach's contraction principle and Precup [13] for other abstract principles (Schauder's, Leray-Schauder's and Krasnoselskii's cone theorems), we may expect that better results can be obtained for system (1.1) if $X^{2}$ is endowed with a family of vectorvalued pseudo-metrics. Of course, in this situation the contraction condition has to be expressed in terms of a matrix instead of scalar Lipschitz constants allowing the two mappings $A$ and $B$ to satisfy more relaxed Lipschitz conditions.

Our first goal in this paper is to present Perov type fixed point theorems for contractive mappings in Gheorghiu's sense on spaces endowed with a family of vector-valued pseudo-metrics. Then we present applications to system (1.2) with parameter standardization $\tau_{1}=\tau_{2}=1$ in two cases:

(a) for advanced arguments $\sigma_{1}(t)=\sigma_{2}(t)=t+2$;

(b) for unmodified arguments $\sigma_{1}(t)=\sigma_{2}(t)=t$.

The use of a gauge structure is motivated by our interest in discussing long term behavior of the system, i.e., $t \in[0, \infty)$, while the advanced arguments in the first example lead to Gheorghiu's contraction notion. Our abstract results are new and complement the existing literature in fixed point theory in gauge/uniform spaces. In addition, compared to previous applications in Precup [14] and Precup-Viorel [15], our new applications give to the vector approach a new asset for its use in the treatment of systems.

In order to make clear the connection of our results to the existing literature, we conclude this introductory section recalling some definitions and results (details can be found in Precup [12]).

By a vector-valued metric on a set $X$ one means a map $d: X \times X \rightarrow \mathbb{R}^{n}$ with the following properties: $d(x, y) \geq 0$ for all $x, y \in X$ and if $d(x, y)=0$ then $x=y$; $d(x, y)=d(y, x)$ for all $x, y \in X ; d(x, y) \leq d(x, z)+d(z, y)$ for all $x, y, z \in X$. 
Here, if $a=\left(a_{1}, a_{2}, \ldots, a_{n}\right), b=\left(b_{1}, b_{2}, \ldots, b_{n}\right) \in \mathbb{R}^{n}$, then by $a \leq b$ we mean that $a_{i} \leq b_{i}$ for $i=1,2, \ldots, n$. A set $X$ endowed with a vector-valued metric $d$ is said to be a generalized metric space. For the generalized metric spaces, the notions of a convergent sequence, Cauchy sequence and completeness are similar to those for usual metric spaces.

Let $(X, d)$ be a generalized metric space. A map $T: X \rightarrow X$ is said to be a generalized contraction if there exists a matrix $M \in \mathbb{M}_{n \times n}\left(\mathbb{R}_{+}\right)$such that

$$
M^{k} \rightarrow 0 \quad \text { as } \quad k \rightarrow \infty
$$

and

$$
d(T(x), T(y)) \leq M \mathrm{~d}(x, y) \quad \text { for all } \quad x, y \in X .
$$

The Lipschitz matrix $M$ satisfying (1.4) is said to be convergent to zero.

The extension of Banach's fixed point theorem to generalized contractions on spaces with a vector-valued metric is due to Perov and Kibenko (see [1] and [12]).

TheOREM 1.1 (Perov). Let $(X, d)$ be a complete generalized metric space and $T: X \rightarrow X$ be a generalized contraction with Lipschitz matrix $M$. Then $T$ has a unique fixed point $x^{*}$ and for each $x_{0} \in X$, one has

$$
\left.d\left(T^{k}\left(x_{0}\right), x^{*}\right)\right) \leq M^{k}(I-M)^{-1} d\left(x_{0}, T\left(x_{0}\right)\right) \quad \text { for all } k \in \mathbb{N} .
$$

As concerns matrices which are convergent to zero, we mention the following equivalent characterizations (see Precup [13]): If $M$ be a square matrix of nonnegative numbers, then the following statements are equivalent:

(i) the matrix $M$ is convergent to zero;

(ii) $I-M$ is non-singular and $(I-M)^{-1}=I+M+M^{2}+\ldots$;

(iii) $|\lambda|<1$ for every $\lambda \in \mathbb{C}$ with $\operatorname{det}(M-\lambda I)=0$;

(iv) $I-M$ is non-singular and $(I-M)^{-1}$ has nonnegative elements.

Finally we recall basic definitions and results of the theory of gauge spaces.

A map $d: X \times X \rightarrow \mathbb{R}_{+}$is said to be a pseudo-metric, or a gauge on the set $X$, if it has the following properties: $d(x, x)=0, d(x, y)=d(y, x)$ and $d(x, y) \leq d(x, z)+d(z, y)$ for all $x, y, z \in X$. A family $\mathcal{P}=\left\{d_{\alpha}\right\}_{\alpha \in \Lambda}$ of pseudometrics on $X$ (or a gauge structure on $X$ ) is said to be separating if for each pair of points $x, y \in X$ with $x \neq y$, there is a $d_{\alpha} \in \mathcal{P}$ such that $d_{\alpha}(x, y) \neq 0$. A pair $(X, \mathcal{P})$ of a nonempty set $X$ and a separating gauge structure $\mathcal{P}$ on $X$ is called a gauge space.

It is well-known (see Dugundji [5] pp. 198-204]) that any family $\mathcal{P}$ of pseudometrics on a set $X$ induces on $X$ a structure $\mathcal{U}$ of uniform space and conversely, any uniform structure on $X$ is induced by a family of pseudo-metrics on $X$. In addition, $\mathcal{U}$ is separating (or Hausdorff) if and only if $\mathcal{P}$ is separating. Hence we may identify gauge spaces to Hausdorff uniform spaces.

We now recall the notion of contraction on a gauge space, introduced by Gheorghiu [7] (see also Chiş-Precup [2] and Angelov [1]). Let $(X, \mathcal{P})$ be a gauge 
space with $\mathcal{P}=\left\{d_{\alpha}\right\}_{\alpha \in \Lambda}$. A map $T: D(T) \subset X \rightarrow X$ is a contraction if there exists a function $\varphi: \Lambda \rightarrow \Lambda$ and $a \in \mathbb{R}_{+}^{\Lambda}, a=\left\{a_{\alpha}\right\}_{\alpha \in \Lambda}$ such that

$$
d_{\alpha}(T(x), T(y)) \leq a_{\alpha} d_{\varphi(\alpha)}(x, y) \quad \text { for all } \quad \alpha \in \Lambda \text { and } x, y \in D(T)
$$

and

$$
\sum_{i=1}^{\infty} a_{\alpha} a_{\varphi(\alpha)} a_{\varphi^{2}(\alpha)} \ldots a_{\varphi^{i-1}(\alpha)} d_{\varphi^{i}(\alpha)}(x, y)<\infty
$$

for every $\alpha \in \Lambda$ and $x, y \in D(T)$. Here $\varphi^{i}$ is the $i$ th iterate of $\varphi$.

We note that this notion was first introduced by Marinescu [10] in locally convex spaces assuming that $\varphi^{2}=\varphi$ and then by Colojoară [3] in uniform spaces, under the same condition. The case $\varphi=1_{\Lambda}$ (identity) was considered by Tarafdar [16] (see also Frigon [6]). Also note that a somewhat different notion of contraction in a uniform space was defined by Knill [9] in terms of entourages.

TheOREM 1.2. (Gheorghiu 7]) Let $(X, \mathcal{P})$ be a complete gauge space and let $T: X \rightarrow X$ be a contraction. Then $T$ has a unique fixed point which can be obtained by successive approximations starting from any element of $X$.

\section{Main abstract results}

In this section we introduce the notions of a vector-valued pseudo-metric, generalized gauge space and generalized contraction. Then Gheorghiu's theorem is extended for generalized contractions on complete generalized gauge spaces. A second result is concerning with mappings which are contractive in Gheorghiu's sense only on one of its orbits. The results are Perov-Gheorghiu mixtures and have the advantages of both approaches.

Definition 2.1. Let $Z$ be a set. A vector-valued map $D: Z \times Z \rightarrow \mathbb{R}_{+}^{n}$ is said to be a vector-valued pseudo-metric, or a vector-valued gauge on $Z$, if it has the following properties: $D(u, u)=0 ; D(u, v)=D(v, u)$; and $D(u, v) \leq$ $D(u, w)+D(w, v)$ for all $u, v, w \in Z$. Here again, if $a=\left(a_{1}, a_{2}, \ldots, a_{n}\right), b=$ $\left(b_{1}, b_{2}, \ldots, b_{n}\right) \in \mathbb{R}^{n}$, then by $a \leq b$ we mean $a_{i} \leq b_{i}$ for $i=1,2, \ldots, n$.

A family $\mathcal{G}=\left\{D_{\alpha}\right\}_{\alpha \in \Lambda}$ of vector-valued pseudo-metrics on $Z$ (or a generalized gauge structure on $Z$ ) is said to be separating if for each pair of points $u, v \in Z$ with $u \neq v$, there is a $D_{\alpha} \in \mathcal{G}$ such that $D_{\alpha}(u, v) \neq 0$. A pair $(Z, \mathcal{G})$ of a nonempty set $Z$ and a separating generalized gauge structure $\mathcal{G}$ on $Z$ is called a generalized gauge space. For the generalized gauge spaces, the notions of a convergent sequence, Cauchy sequence and completeness are similar to those for usual gauge spaces.

By analogy, we can introduce the vector version of Gheorghiu's notion of contraction. 
Definition 2.2. Let $(Z, \mathcal{G})$ be a generalized gauge space with $\mathcal{G}=\left\{D_{\alpha}\right\}_{\alpha \in \Lambda}$. A map $T: D(T) \subset Z \rightarrow Z$ is a generalized contraction if there exists a function $\varphi: \Lambda \rightarrow \Lambda$ and $M \in \mathbb{M}_{n \times n}\left(\mathbb{R}_{+}\right)^{\Lambda}, M=\left\{M_{\alpha}\right\}_{\alpha \in \Lambda}$ such that

$$
D_{\alpha}(T(u), T(v)) \leq M_{\alpha} D_{\varphi(\alpha)}(u, v) \quad \text { for all } \quad \alpha \in \Lambda \text { and } u, v \in D(T)
$$
and

$$
\sum_{i=1}^{\infty} M_{\alpha} M_{\varphi(\alpha)} M_{\varphi^{2}(\alpha)} \ldots M_{\varphi^{i-1}(\alpha)} D_{\varphi^{i}(\alpha)}(u, v)<\infty
$$

for every $\alpha \in \Lambda$ and $u, v \in D(T)$.

Now the Perov type analog for generalized contractions of Gheorghiu's fixed point theorem reads as follows:

TheOREm 2.1. Let $(Z, \mathcal{G})$ be a complete generalized gauge space and let $T: Z \rightarrow Z$ be a generalized contraction. Then $T$ has a unique fixed point which can be obtained by successive approximations starting from any element of $Z$.

Pr o of. Let $u_{0}$ be an arbitrary element of $Z$. Define a sequence $\left(u_{k}\right)$ by

$$
u_{k+1}=T\left(u_{k}\right), \quad k \in \mathbb{N} .
$$

Then using (2.1) we have

$$
\begin{aligned}
D_{\alpha}\left(u_{k}, u_{k+1}\right) & =D_{\alpha}\left(T\left(u_{k-1}\right), T\left(u_{k}\right)\right) \\
& \leq M_{\alpha} D_{\varphi(\alpha)}\left(u_{k-1}, u_{k}\right) \\
& =M_{\alpha} D_{\varphi(\alpha)}\left(T\left(u_{k-2}\right), T\left(u_{k-1}\right)\right) \\
& \leq M_{\alpha} M_{\varphi(\alpha)} D_{\varphi^{2}(\alpha)}\left(u_{k-2}, u_{k-1}\right) \\
& \vdots \\
& \leq M_{\alpha} M_{\varphi(\alpha)} \ldots M_{\varphi^{k-1}(\alpha)} D_{\varphi^{k}(\alpha)}\left(u_{0}, u_{1}\right)
\end{aligned}
$$

for every $\alpha \in \Lambda$ and $k=1,2, \ldots$. As a consequence we have

$$
\begin{aligned}
D_{\alpha}\left(u_{k}, u_{k+m}\right) & =D_{\alpha}\left(u_{k}, u_{k+1}\right)+\cdots+D_{\alpha}\left(u_{k+m-1}, u_{k+m}\right) \\
& \leq \sum_{n=0}^{m-1} M_{\alpha} M_{\varphi(\alpha)} \ldots M_{\varphi^{k+n-1}(\alpha)} D_{\varphi^{k+n}(\alpha)}\left(u_{0}, u_{1}\right) \\
& =\sum_{i=k}^{k+m-1} M_{\alpha} M_{\varphi(\alpha)} \ldots M_{\varphi^{i-1}(\alpha)} D_{\varphi^{i}(\alpha)}\left(u_{0}, u_{1}\right) .
\end{aligned}
$$

Hence, according to (2.2),$\left(u_{k}\right)$ is a Cauchy sequence. Let $u^{*}$ be its limit. Then, letting $k \rightarrow \infty$ in (2.3) gives $u^{*}=T\left(u^{*}\right)$. For uniqueness, assume that $u_{1}, u_{2}$ are two fixed points of $T$. Then

$$
\begin{aligned}
D_{\alpha}\left(u_{1}, u_{2}\right) & =D_{\alpha}\left(T\left(u_{1}\right), T\left(u_{2}\right)\right) \\
& \leq M_{\alpha} D_{\varphi(\alpha)}\left(u_{1}, u_{2}\right)
\end{aligned}
$$




$$
\begin{aligned}
& \text { ADELA NOVAC }- \text { RADU PRECUP } \\
& \leq M_{\alpha} M_{\varphi(\alpha)} D_{\varphi^{2}(\alpha)}\left(u_{1}, u_{2}\right) \\
& \vdots \\
& \leq M_{\alpha} M_{\varphi(\alpha)} \ldots M_{\varphi^{k-1}(\alpha)} D_{\varphi^{k}(\alpha)}\left(u_{1}, u_{2}\right)
\end{aligned}
$$

and using (2.2) we obtain that $D_{\alpha}\left(u_{1}, u_{2}\right)=0$ for every $\alpha \in \Lambda$. Since family $\mathcal{G}$ is separating we deduce that $u_{1}=u_{2}$.

From the proof of Theorem 2.1 we immediately obtain the following result guaranteeing the existence of a fixed point as limit of the successive approximation sequence which starts from a given element of the space.

TheOREm 2.2. Let $(Z, \mathcal{G})$ be a complete generalized gauge space with $\mathcal{G}=$ $\left\{D_{\alpha}\right\}_{\alpha \in \Lambda}$ and let $T: Z \rightarrow Z$ be a mapping. Assume that there is $u_{0} \in Z$, $C>0, \varphi: \Lambda \rightarrow \Lambda$ and $M \in \mathbb{M}_{n \times n}\left(\mathbb{R}_{+}\right)^{\Lambda}, M=\left\{M_{\alpha}\right\}_{\alpha \in \Lambda}$ such that the following conditions hold:

$$
\begin{gathered}
D_{\alpha}\left(T(u), T(v) \leq M_{\alpha} D_{\varphi(\alpha)}(u, v) \quad \text { for all } \alpha \in \Lambda \text { and } u, v \in Z ;\right. \\
\sum_{i=1}^{\infty} M_{\alpha} M_{\varphi(\alpha)} M_{\varphi^{2}(\alpha)} \cdots M_{\varphi^{i-1}(\alpha)}<\infty \quad \text { for every } \quad \alpha \in \Lambda ; \\
D_{\alpha}\left(u_{0}, T\left(u_{0}\right)\right) \leq C \quad \text { for all } \quad \alpha \in \Lambda .
\end{gathered}
$$

Then $T$ has at least one fixed point which can be obtained by successive approximations starting from $u_{0}$.

Remark 1. Here are some useful particular cases: If there is an integer $p \geq 2$ with $\varphi^{p}=\varphi$, then conditions (2.2) and (2.4) reduce to the assumption that

$$
M_{\varphi(\alpha)} \ldots M_{\varphi^{p-1}(\alpha)} \text { is convergent to zero for every } \alpha \in \Lambda \text {. }
$$

Thus, if $p=2$, that is $\varphi^{2}=\varphi$ (Marinescu's situation), then (2.2) and (2.4) hold if

$$
M_{\varphi(\alpha)} \text { is convergent to zero for every } \alpha \in \Lambda \text {. }
$$

In particular, if $\varphi=1_{\Lambda}$ (Tarafdar's situation), then (2.2) and (2.4) are satisfied provided that

$$
M_{\alpha} \text { is convergent to zero for every } \alpha \in \Lambda \text {. }
$$

Now we turn back to system (1.1). We assume that $X$ is a complete gauge space with the family of pseudo-metrics $\mathcal{P}=\left\{d_{\alpha}\right\}_{\alpha \in \Lambda}$. We denote

$$
Z:=X^{2}, \quad T:=(A, B) \quad \text { and } \quad \mathcal{G}:=\left\{D_{\alpha}\right\}_{\alpha \in \Lambda},
$$

where

$$
D_{\alpha}(u, v)=\left[\begin{array}{l}
d_{\alpha}\left(x, x_{1}\right) \\
d_{\alpha}\left(y, y_{1}\right)
\end{array}\right]
$$

for every $u:=(x, y), v:=\left(x_{1}, y_{1}\right) \in X^{2}$ and $\alpha \in \Lambda$. Then $(Z, \mathcal{G})$ is a complete generalized gauge space.

Specialized to this case, Theorems 2.1 and 2.2 yield the following results. 
TheOREM 2.3. Assume that $(X, \mathcal{P})$ is a complete gauge space with $\mathcal{P}=\left\{d_{\alpha}\right\}_{\alpha \in \Lambda}$ and that there exists a function $\varphi: \Lambda \rightarrow \Lambda$ and nonnegative constants $a_{\alpha}, b_{\alpha}$, $\bar{a}_{\alpha}, \bar{b}_{\alpha}$ such that

$$
\begin{aligned}
& d_{\alpha}\left(A(x, y), A\left(x_{1}, y_{1}\right)\right) \leq a_{\alpha} d_{\varphi(\alpha)}\left(x, x_{1}\right)+b_{\alpha} d_{\varphi(\alpha)}\left(y, y_{1}\right), \\
& d_{\alpha}\left(B(x, y), B\left(x_{1}, y_{1}\right)\right) \leq \bar{a}_{\alpha} d_{\varphi(\alpha)}\left(x, x_{1}\right)+\bar{b}_{\alpha} d_{\varphi(\alpha)}\left(y, y_{1}\right)
\end{aligned}
$$

for all $x, x_{1}, y, y_{1} \in X$ and $\alpha \in \Lambda$. Let

$$
M_{\alpha}:=\left[\begin{array}{ll}
a_{\alpha} & b_{\alpha} \\
\bar{a}_{\alpha} & \bar{b}_{\alpha}
\end{array}\right]
$$

If

$$
\sum_{i=1}^{\infty} M_{\alpha} M_{\varphi(\alpha)} M_{\varphi^{2}(\alpha)} \ldots M_{\varphi^{i-1}(\alpha)} D_{\varphi^{i}(\alpha)}(u, v)<\infty
$$

for all $u, v \in X^{2}$ and $\alpha \in \Lambda$, then system (1.1) has a unique solution. Moreover, the solution is the limit of the sequence of successive approximations

$$
u_{k}=\left(x_{k}, y_{k}\right), \quad x_{k+1}=A\left(x_{k}, y_{k}\right), \quad y_{k+1}=B\left(x_{k}, y_{k}\right) \quad(k=0,1, \ldots)
$$

starting from any initial pair $\left(x_{0}, y_{0}\right) \in X^{2}$.

P r o of. Clearly inequalities (2.6) can be written in the vector form

$$
D_{\alpha}(T(u), T(v)) \leq M_{\alpha} D_{\varphi(\alpha)}(u, v) .
$$

The result is now a direct consequence of Theorem 2.1 .

THEOREM 2.4. Under the assumptions of Theorem 2.3, if there is $u_{0}=\left(x_{0}, y_{0}\right)$ $\in X^{2}$ and $C>0$ such that

$$
D_{\alpha}\left(u_{0}, T\left(u_{0}\right)\right) \leq C
$$

and

$$
\sum_{i=1}^{\infty} M_{\alpha} M_{\varphi(\alpha)} M_{\varphi^{2}(\alpha)} \ldots M_{\varphi^{i-1}(\alpha)}<\infty
$$

for every $\alpha \in \Lambda$, then system (1.1) has at least one solution which is the limit of sequence (2.8) starting from $u_{0}$.

Pr o of. The result is a direct consequence of Theorem 2.2 . 


\section{Applications to integral systems}

\subsection{A system of integral equations with advanced argument}

Consider the system of integral equations with advanced argument

$$
\left\{\begin{array}{l}
x(t)=\int_{t-1}^{t} f(s, x(s+2), y(s+2)) \mathrm{d} s \\
y(t)=\int_{t-1}^{t} g(s, x(s+2), y(s+2)) \mathrm{d} s
\end{array}\right.
$$

for $t \in[0, \infty)$.

Assume that

$$
\begin{gathered}
\left|f(t, x, y)-f\left(t, x_{1}, y_{1}\right)\right| \leq k_{1}(t)\left|x-x_{1}\right|+k_{2}(t)\left|y-y_{1}\right| \\
\left|g(t, x, y)-g\left(t, x_{1}, y_{1}\right)\right| \leq k_{3}(t)\left|x-x_{1}\right|+k_{4}(t)\left|y-y_{1}\right|
\end{gathered}
$$

for every $x, x_{1}, y, y_{1} \in \mathbb{R}, t \in[-1, \infty)$ and some $k_{i} \in L^{1}\left([-1, \infty), \mathbb{R}_{+}\right), i=$ $1,2,3,4$. For each $n \in \mathbb{N}$, let

$$
\begin{array}{ll}
a_{n}=\int_{n-1}^{2 n+1} k_{1}(t) \mathrm{d} t, \quad b_{n}=\int_{n-1}^{2 n+1} k_{2}(t) \mathrm{d} t, \\
\bar{a}_{n}=\int_{n-1}^{2 n+1} k_{3}(t) \mathrm{d} t, \quad \bar{b}_{n}=\int_{n-1}^{2 n+1} k_{4}(t) \mathrm{d} t
\end{array}
$$

and consider the matrix

Also define the matrix $M_{\infty}$ by

$$
M_{n}=\left[\begin{array}{ll}
a_{n} & b_{n} \\
\bar{a}_{n} & \bar{b}_{n}
\end{array}\right] .
$$

$$
M_{\infty}=\left[\begin{array}{ll}
\left|k_{1}\right|_{L^{1}([-1, \infty))} & \left|k_{2}\right|_{L^{1}([-1, \infty))} \\
\left|k_{3}\right|_{L^{1}([-1, \infty))} & \left|k_{4}\right|_{L^{1}([-1, \infty))}
\end{array}\right] .
$$

Our main result on system (3.1) is the following theorem.

TheOREM 3.1. Let $f, g:[-1, \infty) \times \mathbb{R}^{2} \rightarrow \mathbb{R}$ be two continuous functions and assume that inequalities (3.2) hold for some $k_{i} \in L^{1}\left([-1, \infty), \mathbb{R}_{+}\right), i=1,2,3,4$. In addition assume that there is $u_{0}=\left(x_{0}, y_{0}\right) \in C\left([0, \infty), \mathbb{R}^{2}\right)$ and $C>0$ such that

$$
\left|T\left(u_{0}\right)(t)-u_{0}(t)\right| \leq C \quad \text { for all } t \in[0, \infty),
$$

where $T=(A, B)$ is given bellow. If the matrix

$$
M_{\infty} \text { is convergent to zero, }
$$

then system (3.1) has at least one solution $(x, y) \in C\left([0, \infty), \mathbb{R}^{2}\right)$. 
Pr o of. We shall use Theorem 2.4. Here $X=C[0, \infty), \Lambda=\mathbb{N}$ and for $n \in \mathbb{N}$, $d_{n}: X \times X \rightarrow \mathbb{R}_{+}$is given by

$$
d_{n}(x, y)=\max _{t \in[n, 2 n+1]}|x(t)-y(t)|
$$

Let $A, B: C\left([0, \infty), \mathbb{R}^{2}\right) \rightarrow C[0, \infty)$ be defined by

$$
\begin{aligned}
& A(x, y)(t)=\int_{t-1}^{t} f(s, x(s+2), y(s+2)) \mathrm{d} s, \\
& B(x, y)(t)=\int_{t-1}^{t} g(s, x(s+2), y(s+2)) \mathrm{d} s .
\end{aligned}
$$

First we prove the Lipschitz condition (2.6) with $\varphi: \mathbb{N} \rightarrow \mathbb{N}$ given by $\varphi(n)=n+1$. Let $t \in[n, 2 n+1]$. We have $t-1 \in[n-1,2 n]$, and when $s \in[t-1, t]$, then $s+2 \in[n+1,2 n+3]$. It follows that

$$
\begin{aligned}
& \left|A(x, y)(t)-A\left(x_{1}, y_{1}\right)(t)\right| \\
\leq & \int_{n-1}^{2 n+1}\left|f(s, x(s+2), y(s+2))-f\left(s, x_{1}(s+2), y_{1}(s+2)\right) \mathrm{d} s\right| \\
\leq & \int_{n-1}^{2 n+1} k_{1}(s)\left|x(s+2)-x_{1}(s+2)\right| \mathrm{d} s+\int_{n-1}^{2 n+1} k_{2}(s)\left|y(s+2)-y_{1}(s+2)\right| \mathrm{d} s \\
\leq & \max _{s \in[n-1,2 n+1]}\left|x(s+2)-x_{1}(s+2)\right| \int_{n-1}^{2 n+1} k_{1}(s) \mathrm{d} s \\
& +\max _{s \in[n-1,2 n+1]}\left|y(s+2)-y_{1}(s+2)\right| \int_{n-1}^{2 n+1} k_{2}(s) \mathrm{d} s \\
= & \underset{\tau \in[n+1,2 n+3]}{\max _{2 n+1}\left|x(\tau)-x_{1}(\tau)\right| \int_{n-1}^{2 n+1} k_{1}(s) \mathrm{d} s} \\
& +\max _{\tau \in[n+1,2 n+3]}\left|y(\tau)-y_{1}(\tau)\right| \int_{n-1} k_{2}(s) \mathrm{d} s \\
= & a_{n} d_{n+1}\left(x, x_{1}\right)+b_{n} d_{n+1}\left(y, y_{1}\right) .
\end{aligned}
$$

Taking the maximum over $[n, 2 n+1]$ yields

$$
d_{n}\left(A(x, y), A\left(x_{1}, y_{1}\right)\right) \leq a_{n} d_{n+1}\left(x, x_{1}\right)+b_{n} d_{n+1}\left(y, y_{1}\right)
$$

for every $(x, y),\left(x_{1}, y_{1}\right) \in X^{2}$. 
Similarly, for $B$,

$$
d_{n}\left(B(x, y), B\left(x_{1}, y_{1}\right)\right) \leq \bar{a}_{n} d_{\varphi(n)}\left(x, x_{1}\right)+\bar{b}_{n} d_{\varphi(n)}\left(y, y_{1}\right)
$$

for every $(x, y),\left(x_{1}, y_{1}\right) \in X^{2}$. Hence (2.6) holds. Furthermore, condition (2.9) is guaranteed by assumption (3.3). Also, for every $n \in \mathbb{N}, M_{n} \leq M_{\infty}$ and thus series (2.10) is dominated by

$$
\sum_{k=0}^{\infty} M_{\infty}^{k}
$$

which is convergent in view of assumption (3.4). Hence (2.10) is satisfied. Therefore Theorem 2.4 can be applied.

\subsection{An integral system without modification of the argument}

Consider the system of integral equations

$$
\left\{\begin{array}{l}
x(t)=\int_{t-1}^{t} f(s, x(s), y(s)) \mathrm{d} s \\
y(t)=\int_{t-1}^{t} g(s, x(s), y(s)) \mathrm{d} s
\end{array}\right.
$$

for $t \in[0, \infty)$, where

$$
x(t)=\psi(t) \quad \text { and } \quad y(t)=\phi(t) \quad \text { for } \quad t \in[-1,0]
$$

and $\psi, \phi$ are two given functions.

We assume that inequalities (3.2) hold for every $x, x_{1}, y, y_{1} \in \mathbb{R}, t \in[0, \infty)$ and some $k_{i} \in L_{l o c}^{1}\left([0, \infty), \mathbb{R}_{+}\right), i=1,2,3,4$. For $n \in \mathbb{N} \backslash\{0\}$, we denote

$$
\begin{array}{ll}
a_{n}=\int_{0}^{n} k_{1}(t) \mathrm{d} t, \quad b_{n}=\int_{0}^{n} k_{2}(t) \mathrm{d} t, \\
\bar{a}_{n}=\int_{0}^{n} k_{3}(t) \mathrm{d} t, \quad \bar{b}_{n}=\int_{0}^{n} k_{4}(t) \mathrm{d} t .
\end{array}
$$

and we consider the matrix

$$
M_{n}=\left[\begin{array}{ll}
a_{n} & b_{n} \\
\bar{a}_{n} & \bar{b}_{n}
\end{array}\right] .
$$

TheOrem 3.2. Let $f, g:[-1, \infty) \times \mathbb{R}^{2} \rightarrow \mathbb{R}$ be two continuous functions, $\phi, \psi \in$ $C[-1,0]$ and assume that inequalities (3.2) hold for some $k_{i} \in L_{\text {loc }}^{1}\left([0, \infty), \mathbb{R}_{+}\right)$, $i=1,2,3,4$. If for every $n \in \mathbb{N} \backslash\{0\}$, matrix

$$
M_{n} \text { is convergent to zero, }
$$

then system (3.6) has a unique solution $(x, y) \in C\left([0, \infty), \mathbb{R}^{2}\right)$. 
Pr o of. The result follows from Theorem 2.3 if we take into account Remark 1 about Tarafdar's situation. Here $X=C[0, \infty), \Lambda=\mathbb{N} \backslash\{0\}$, for each $n \in \mathbb{N} \backslash\{0\}$, $d_{n}: X \times X \rightarrow \mathbb{R}_{+}$is given by

$$
d_{n}(x, y)=\max _{t \in[0, n]}|x(t)-y(t)|
$$

$\varphi: \mathbb{N} \backslash\{0\} \rightarrow \mathbb{N} \backslash\{0\}, \varphi(n)=n$, and $A, B: C\left([0, \infty), \mathbb{R}^{2}\right) \rightarrow C[0, \infty)$ are defined by

$$
\begin{aligned}
& A(x, y)(t)=\int_{t-1}^{t} f(s, \tilde{x}(s), \tilde{y}(s)) \mathrm{d} s, \\
& B(x, y)(t)=\int_{t-1}^{t} g(s, \tilde{x}(s), \tilde{y}(s)) \mathrm{d} s,
\end{aligned}
$$

where

$$
\tilde{x}(t)=\left\{\begin{array}{ll}
\psi(t) & \text { for }-1 \leqslant t<0 \\
x(t) & \text { for } t \geqslant 0,
\end{array} \quad \tilde{y}(t)= \begin{cases}\phi(t) & \text { for }-1 \leqslant t<0 \\
y(t) & \text { for } t \geqslant 0\end{cases}\right.
$$

Remark 2. When $k_{i} \in L^{1}\left([0, \infty), \mathbb{R}_{+}\right), i=1,2,3,4$, then a sufficient condition for (3.7) to hold for every $n \in \mathbb{N} \backslash\{0\}$ is that the matrix

$$
M_{\infty}:=\left[\begin{array}{ll}
\left|k_{1}\right|_{L^{1}\left(\mathbb{R}_{+}\right)} & \left|k_{2}\right|_{L^{1}\left(\mathbb{R}_{+}\right)} \\
\left|k_{3}\right|_{L^{1}\left(\mathbb{R}_{+}\right)} & \left|k_{4}\right|_{L^{1}\left(\mathbb{R}_{+}\right)}
\end{array}\right] \quad \text { is convergent to zero. }
$$

Indeed, for each $n \in \mathbb{N} \backslash\{0\}$, one has $M_{n} \leq M_{\infty}$, whence, since the entries of all matrices are nonnegative, $M_{n}^{k} \leq M_{\infty}^{k}$ for all $k \in \mathbb{N}$. Consequently, if $M_{\infty}^{k} \rightarrow 0$ as $k \rightarrow \infty$, then $M_{n}^{k} \rightarrow 0$ as $k \rightarrow \infty$, too. However, (3.8) is not a necessary condition for (3.7) as shows the following contre-example:

$$
k_{1}(t)=(t+1)^{-2}, \quad k_{2}=k_{3}=k_{4}=0 .
$$

In this case

$$
M_{n}=\left[\begin{array}{cc}
\frac{n}{n+1} & 0 \\
0 & 0
\end{array}\right] \quad \text { and } \quad M_{\infty}=\left[\begin{array}{ll}
1 & 0 \\
0 & 0
\end{array}\right] .
$$

Clearly, for every $n \in \mathbb{N} \backslash\{0\}, M_{n}$ converges to zero, but $M_{\infty}$ does not.

Acknowledgement. The authors thank Professor Mircea Ivan for having clarified the situation described by Remark 2 . 


\section{ADELA NOVAC — RADU PRECUP}

\section{REFERENCES}

[1] ANGELOV, V. G.: Fixed Points in Uniform Spaces and Applications, Cluj University Press, Cluj-Napoca, 2009.

[2] CHIS, A.-PRECUP, R.: Continuation theory for general contractions in gauge spaces, Fixed Point Theory Appl. 2004 (2004), 173-185.

[3] COLOJOARĂ, I.: Sur un théorème de point fixe dans les espaces uniformes complets, Com. Acad. R. P. Rom. 11 (1961), 281-283.

[4] COOKE, K. L.-KAPLAN, J. L.: A periodicity threshold theorem for epidemics and population growth, Math. Biosci. 31 (1976), 87-104.

[5] DUGUNDJI, J.: Topology, Allyn and Bacon, Massachusetts, 1966.

[6] FRIGON, M.: Fixed point and continuation results for contractions in metric and gauge spaces. In: Fixed Point Theory and Its Applications. Banach Center Publ. 77, Polish Acad. Sci., Warsaw, 2007, pp. 89-114.

[7] GHEORGHIU, N.: Contraction theorem in uniform spaces, Stud. Cerc. Mat. 19 (1967), 119-122 (Romanian).

[8] GHEORGHIU, N.: Fixed point theorems in uniform spaces, An. Ştiinţ. Univ. Al. I. Cuza Iaşi. Secţ. I Mat. 28 (1982), 17-18.

[9] KNILL, R. J.: Fixed points of uniform contractions, J. Math. Anal. Appl. 12 (1965), 449-455.

[10] MARINESCU, G.: Topological and Pseudo topological Vector Spaces, Editura Acad. R. P. Rom., Bucharest, 1959 (Romanian).

[11] PEROV, A. I.-KIBENKO, A. V.: On a certain general method for investigation of boundary value problems, Izv. Akad. Nauk SSSR 30 (1966), 249-264 (Russian).

[12] PRECUP, R.: Methods in Nonlinear Integral Equations, Kluwer, Dordrecht, 2002.

[13] PRECUP, R.: The role of matrices that are convergent to zero in the study of semilinear operator systems, Math. Comput. Modelling 49 (2009), 703-708.

[14] PRECUP, R.: Existence, localization and multiplicity results for positive radial solutions of semilinear elliptic systems, J. Math. Anal. Appl. 352 (2009), 48-56.

[15] PRECUP, R.-VIOREL, A.: Existence results for systems of nonlinear evolution inclusions, Fixed Point Theory 11 (2010), 337-346.

[16] TARAFDAR, E.: An approach to fixed-point theorems on uniform spaces, Trans. Amer. Math. Soc. 191 (1974), 209-225.

Received 26. 11. 2011

Accepted 1. 5. 2012

\author{
* Department of Mathematics \\ Technical University of Cluj-Napoca \\ 26-28 George Baritiu Street \\ 400027 Cluj-Napoca \\ ROMANIA \\ E-mail: adela.chis@math.utcluj.ro \\ ** Department of Mathematics \\ Babeş-Bolyai University \\ 1 Mihail Kogălniceanu Street \\ 400084 Cluj-Napoca \\ ROMANIA \\ E-mail: r.precup@math.ubbcluj.ro
}

